\title{
Resolution of severely impaired cognitive function following medical treatment of cystic invasive
}

macroprolactinoma

Bukowczan J, Lois K, Mathiopoulou M, Kelly T, Abouglila K, Mitra D, James RA, Regional Pituitary Tumour Service, Newcastle upon Tyne Hospitals NHS Foundation Trust, Newcastle upon Tyne , United Kingdom.

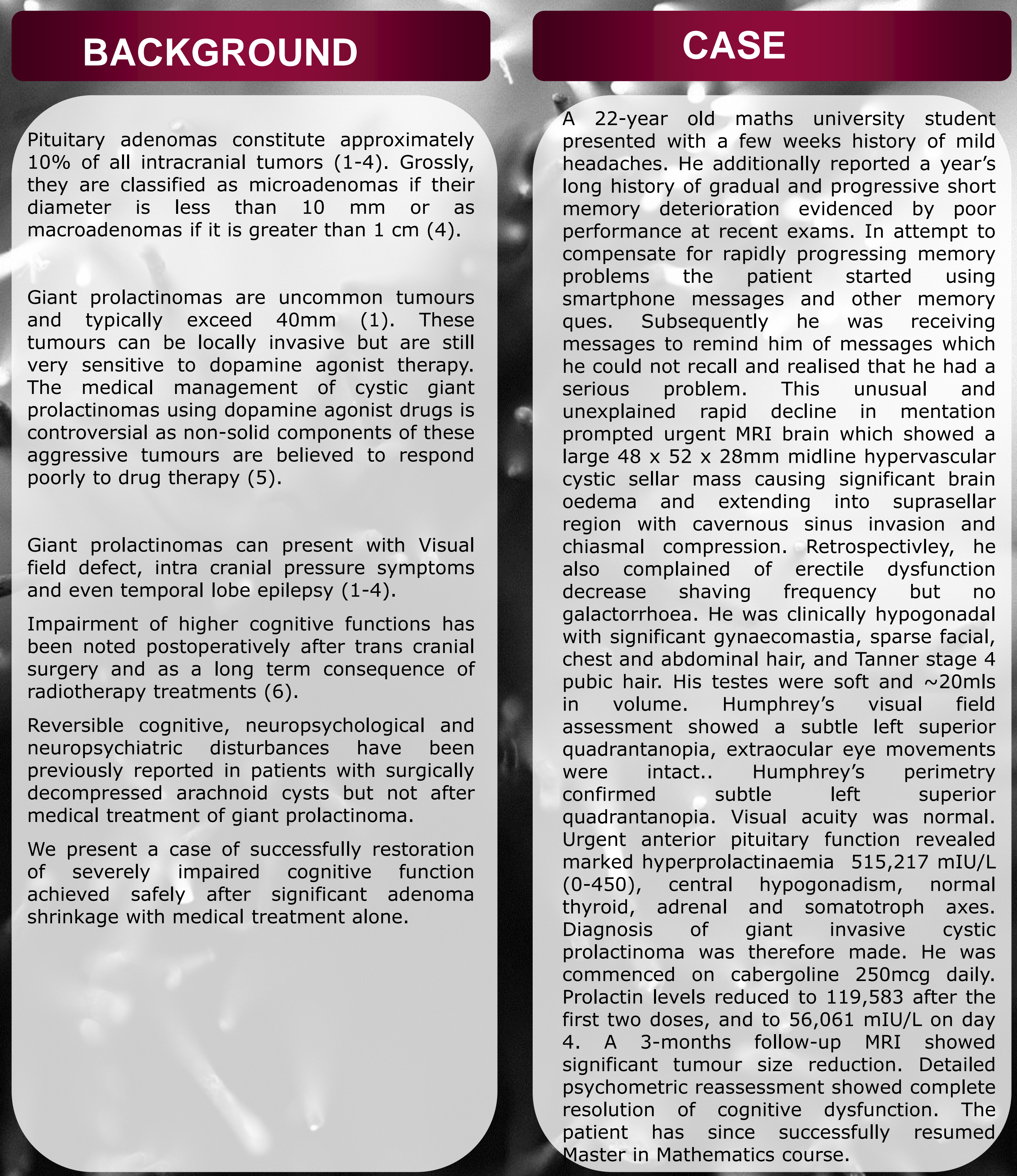

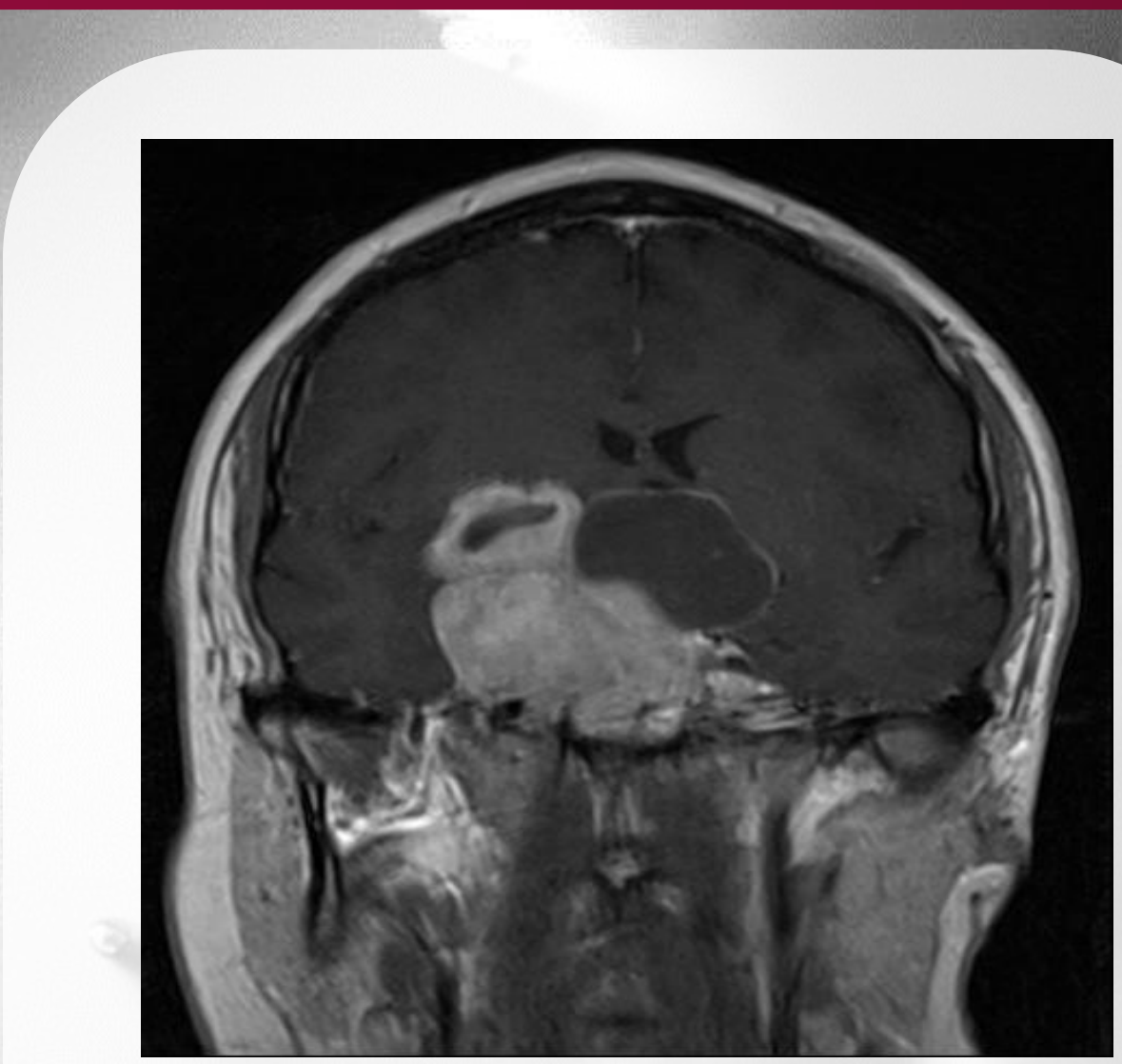

Fig. 1 MRI Pituitary at presentation

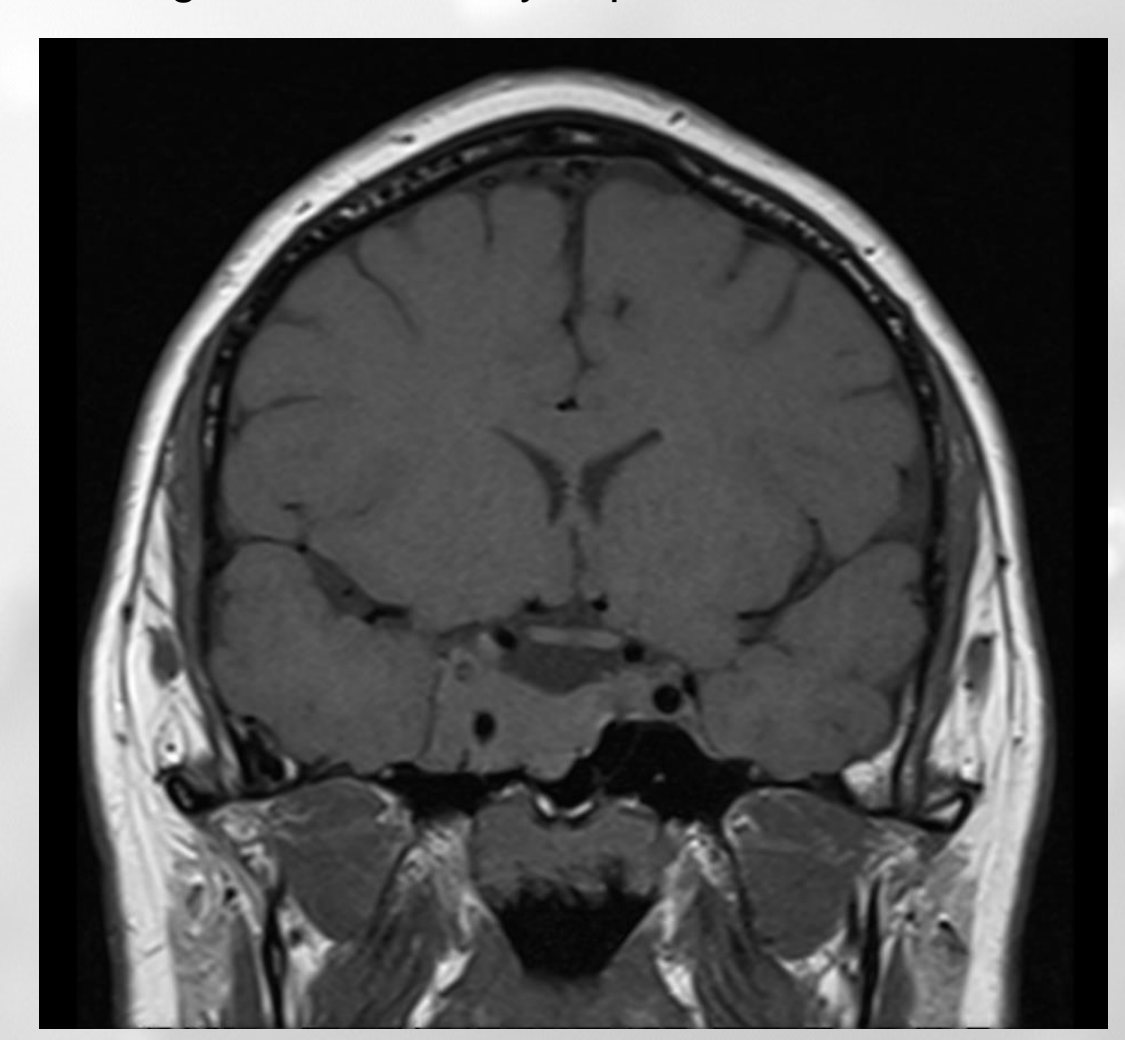

Fig. 2 MRI Pituitary following 6

months of cabergoline treatment

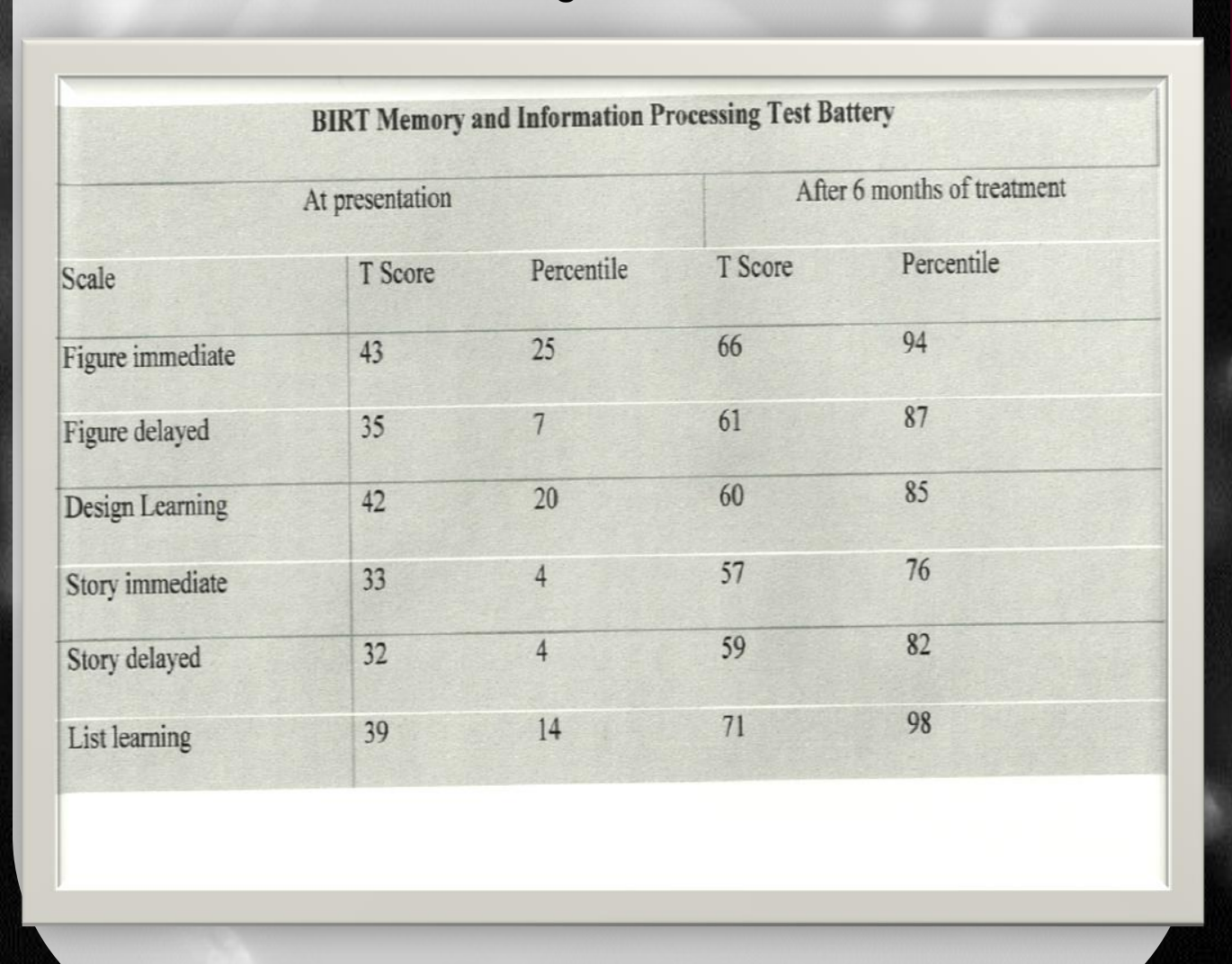

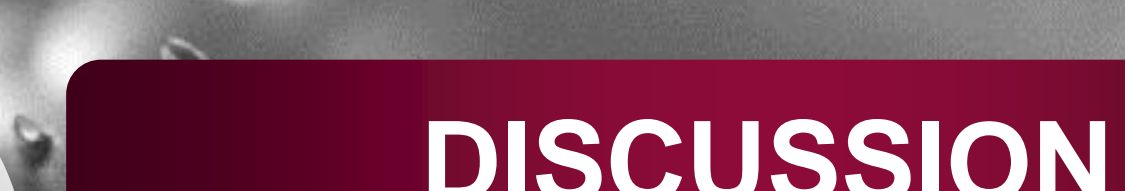

Reversible dyscognition with severe short term memory loss and personality disorders were reported in patients with surgically decompressed extracerebral cysts. Cystic giant prolactinomas can masquerade other intracranial malignancies but surgical intervention should be avoided. Dopamine agonists remains first line treatment obviating risks of pituitary surgery. This case highlights effectiveness of medical management in giant prolactinoma and is the first to report dramatic resolution of debilitating cognitive impairment.

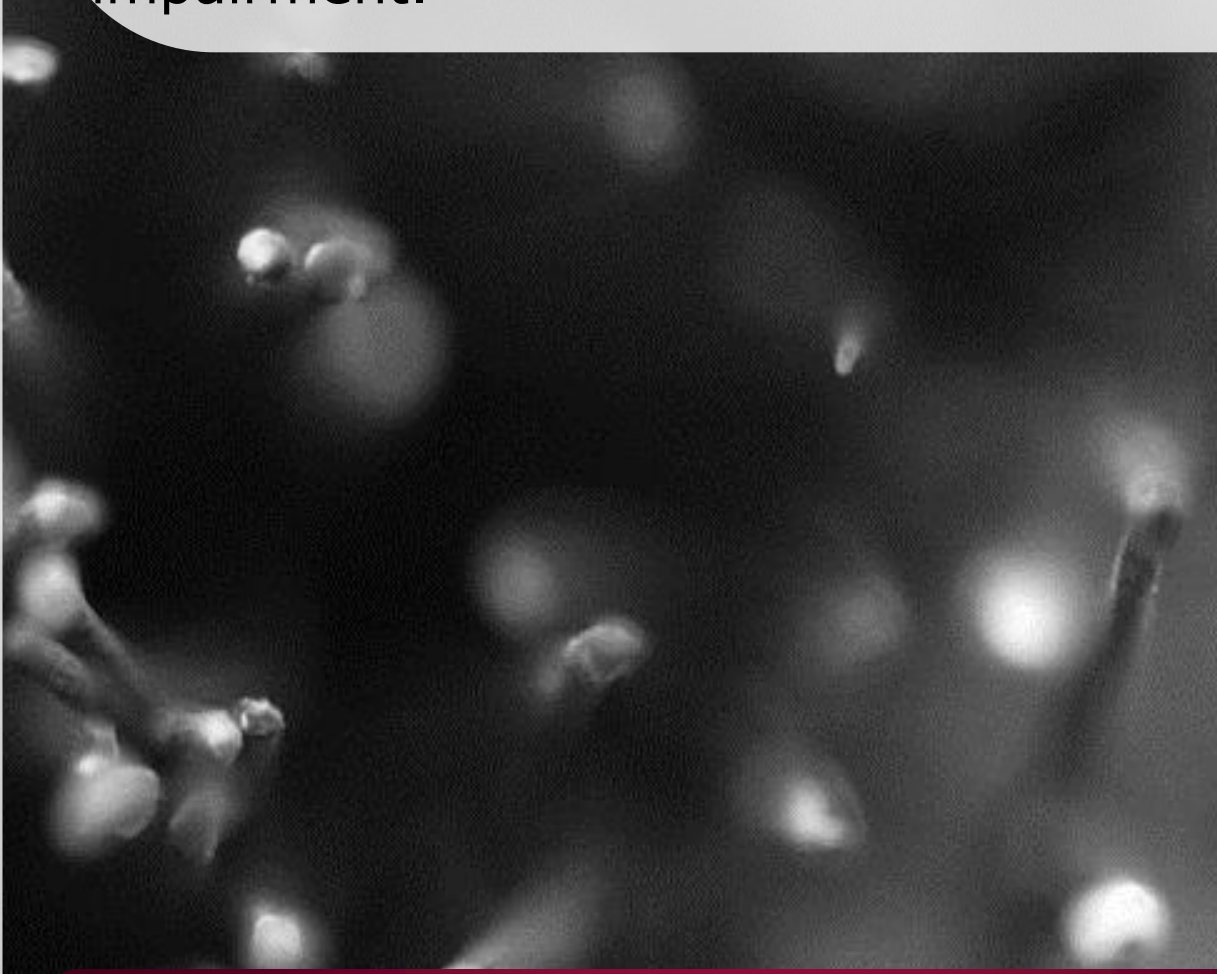

REFERENCES

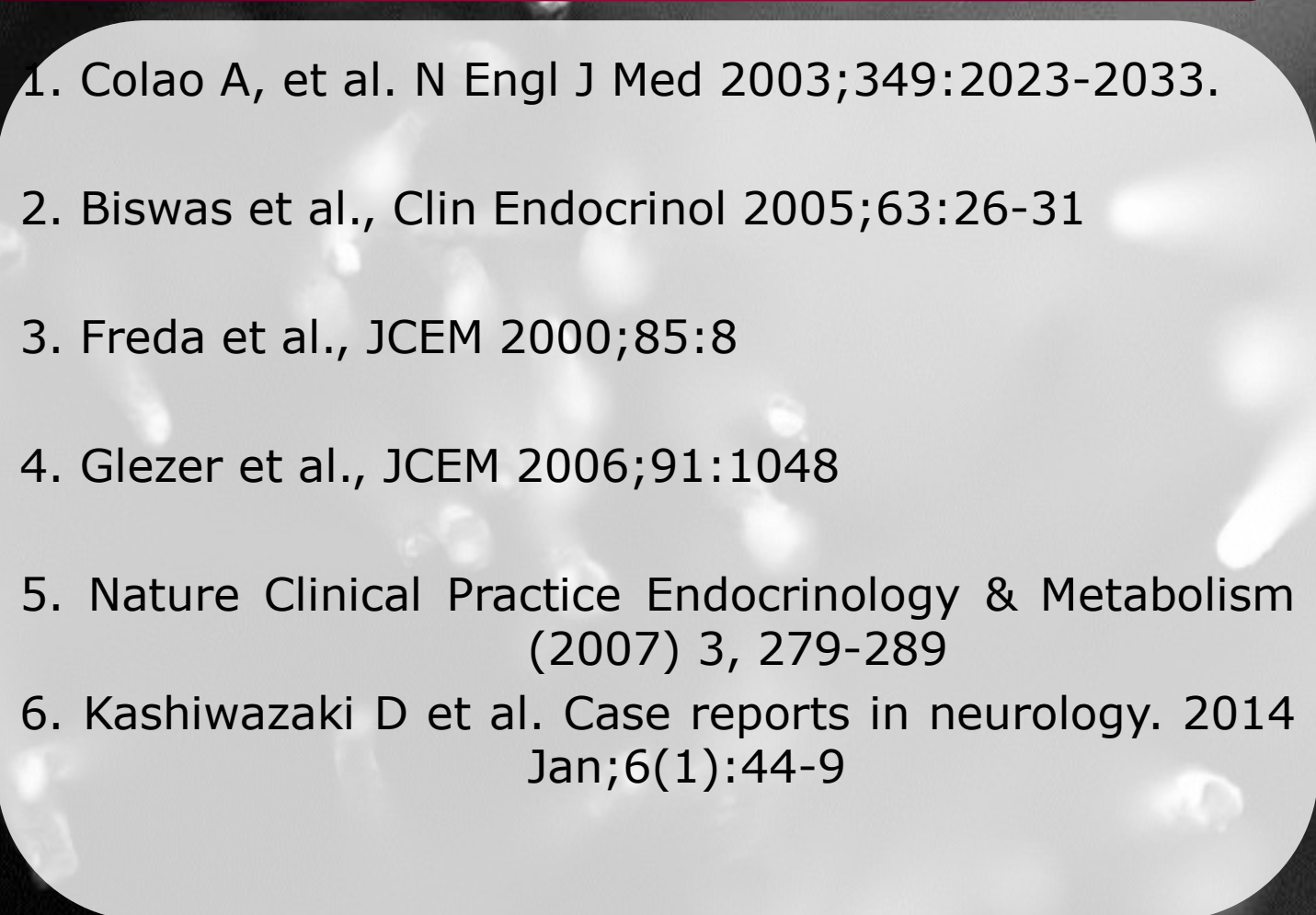

